\title{
Efficience Du Profit Des Micro-Entreprises Camerounaises Et Réduction De La Pauvreté Monétaire : Une Analyse Par Segmentation
}

\author{
Tsachoung Jean-Marie \\ Doctorant à l'Université de Dschang, Cameroon \\ Faculté des Sciences Economique et de Gestion
}

Kana Kenfack Christophe

Institut National de la Statistique

Doi:10.19044/esj.2019.v15n4p374～㞷L:http://dx.doi.org/10.19044/esj.2019.v15n4p374

Résumé

L'objectif de cette étude est de déterminer la contribution de l'efficience du profit dans la réduction de la pauvreté des micro-entrepreneurs et d'identifier les facteurs explicatifs du niveau d'efficience. En revisitant les travaux existants, nous avons proposé pour le cas des micro-entreprises camerounaises une approche paramétrique pour mesurer le niveau d'efficience. Pour prendre en compte l'hétérogénéité de ces micro-entreprises, nous avons procédé à la segmentation de celles-ci par une méthode de mélange de modèles de régression. L'estimation économétrique de ce modèle nous a permis de retenir deux segments des micro-entreprises camerounaises. Un segment constitué de très petites micro-entreprises et un deuxième formé des micro-entreprises proches des entreprises du secteur formel. La méthode paramétrique utilisée a été confirmée par l'estimation des frontières de profit dans chaque segment et pour l'ensemble des micro-entreprises où les deux termes d'erreur ont été significativement non nuls. L'évaluation de l'impact de la situation d'efficience s'est faite en comparant les niveaux de pauvreté obtenus à partir des revenus réels avec ceux obtenus en utilisant les profits d'efficience. Comme résultat, il ressort qu'en situation d'efficience, $10,9 \%$ des micro-entrepreneurs migreront de la situation de pauvreté à celle de non pauvreté. Ce taux équivaut à $11,5 \%$ des micro-entrepreneurs du premier segment et $3,1 \%$ de ceux du deuxième segment. Concernant les facteurs explicatifs, il ressort qu'en considérant les micro-entreprises du premier segment et toutes les micro-entreprises dans l'ensemble, le niveau d'efficience est positivement lié aux facteurs de positionnement, facteurs liés aux promoteurs et aux facteurs liés à la micro-entreprise. 
Mots clés: Micro-entreprise, efficience, frontière stochastique de profit, pauvreté

\title{
Profit Efficiency of Micro-Enterprises and Poverty Reduction in Cameroon: A Segmentation Analysis Approach
}

\author{
Tsachoung Jean-Marie \\ Doctorant à l'Université de Dschang, Cameroon \\ Faculté des Sciences Economique et de Gestion
}

Kana Kenfack Christophe

Institut National de la Statistique

\begin{abstract}
Abstrat
The objective of this study is to determine the contribution of profit efficiency in poverty reduction of micro-entrepreneurs and to identify factors explaining the level of efficiency. In view of existing works, we proposed in the case of Cameroonian micro-enterprises a parametric approach to measure the level of efficiency. Considering their heterogeneity, we preceded their segmentation by mixtures of regression models. The impact assessment of the efficiency level is made by comparing poverty levels obtained from the real incomes with those obtained using efficiency revenue.

As a result, in a situation efficiency, $10.9 \%$ micro-entrepreneurs will migrate from poverty to a state of non- poverty. Concerning the explanatory factors, level of efficiency of micro- enterprises of first segment and all microenterprises as a whole is positively related to environment, microentrepreneurs and their micro-enterprises. This is equivalent to $11.5 \%$ of micro-entrepreneurs in the first segment and $3.1 \%$ of those in the second segment.
\end{abstract}

Keywords: Micro-enterprise, efficiency, stochastic profit frontier, poverty

\section{Introduction}

Le Cameroun s'est présenté jusqu'au début des années 1980 comme l'un des rares pays en Afrique Sub-saharienne à avoir le moins souffert des effets de la crise pétrolière (1973-1974). Après ces années de relative prospérité (après l'indépendance jusqu'au début des années 1980), son économie a été confrontée à une profonde crise économique (1985-1994) 
marquée par des déséquilibres macroéconomiques, l'effondrement des revenus des ménages et la détérioration des conditions de vie des populations (INS ,2002). Face à cette situation, le Cameroun a adopté le Programme $\mathrm{d}^{\prime}$ Ajustement Structurel (PAS) ${ }^{7}$ visant à redresser son économie. Comme dans plusieurs pays en voie de développement, les résultats escomptés n'ont pas été atteints. On a assisté à une augmentation de la pauvreté et une mauvaise distribution de revenu (Baden, 1997). En 1996, plus de 50\% des Camerounais vivaient en dessous du seuil de pauvreté et le taux d'informalisation s'élevait à $85,9 \%$ (INS, 1996)

Depuis 1994, suite à la dévaluation ${ }^{8}$ du FCFA et à de nombreux programmes ${ }^{9}$ entrepris, l'économie camerounaise a repris le chemin de la croissance. Cependant, la réduction de la pauvreté n'a pas été significative. En effet, il ressort des Enquêtes Camerounaises Auprès des Ménages $(\text { ECAM })^{10}$ qu'entre 2001 et 2007 , le taux de pauvreté n'a reculé que de 0,3 point, passant ainsi de 40,2 à 39,9\%. Ceci indique clairement l'échec du gouvernement camerounais à réaliser l'objectif inscrit dans le Document de Stratégie de Réduction de la Pauvreté (DSRP) qui est la réduction de la pauvreté à 37\% (Manga et Epo ,2009). La croissance est ainsi nécessaire mais pas suffisante pour combattre la pauvreté. Il est donc important de chercher des stratégies de croissance favorables aux pauvres. Selon Lachaud (1999), le succès de toute politique de développement visant à réduire la pauvreté à court terme et à l'éradiquer à long terme est étroitement lié au mode de fonctionnement du marché du travail. Pour Pineau (2004), l'amélioration de la productivité dans le marché du travail est ce dont les pauvres ont besoin pour sortir de la pauvreté. Le marché du travail du fait qu'il constitue la principale source de revenu de la plupart des ménages serait donc la cible pour combattre la pauvreté. Au Cameroun, ce marché est caractérisé par une prépondérance du secteur informel. Il ressort des rapports publiés dans le cadre de l'enquête EESI(2010) qu'environ 90,5\% des emplois sont informels. Ces rapports mettent également en exergue l'inefficience des micro-entreprises que constitue ce secteur. Ces micro-entreprises sont caractérisées par une gestion précaire de leurs activités, un rendement économique qui ne permet pas toujours au promoteur de vivre au-dessus du seuil de pauvreté. Outre cette précarité, le sous -emploi y est important et touche près de trois quarts des

7Ces programmes d'Ajustement Structurels (PAS) préconisaient entre autres, la compression des dépenses publiques afin de réduire les déficits budgétaires ; ce qui a fortement affecté le marché du travail et il s'en est suivi le blocage des salaires et même parfois la réduction des salaires des travailleurs

8 Le Franc CFA a été dévalué de 50\% par rapport au Franc Français en accord avec les autres pays de la zone Franc

9 On peut mentionner le programme économique et financier (programme triennal)

${ }^{10}$ L'enquête ECAM II a été réalisée en 2001 et ECAM III en 2007. 
actifs. Sachant que près de $90 \%$ des emplois sont informels pendant que plus de $40 \%$ des ménages camerounais vivent en dessous du seuil de pauvreté avec l'emploi informel comme principal source de revenu, il convient de se poser la question suivante : Que gagnerait-on en termes de réduction de la pauvreté à porter les micro-entreprises à l'efficience et quels sont les facteurs influençant cette efficience?

Pour répondre à cette question nous nous sommes fixés pour objectifs de : mesurer la proportion des micro-entrepreneurs qui une fois à l'efficience ont des revenus leur permettant de vivre au-dessus du seuil de pauvreté et d'identifier les facteurs qui pourraient contribuer à l'amélioration du niveau d'efficience. La notion d'efficience bien connue dans le secteur informel désigne l'écart qui existe entre la production d'un micro-entrepreneur par rapport à celle qui est techniquement possible, ce niveau de possibilité étant déterminé au regard des performances des autres producteurs similaires.

Le reste de cette étude s'articule autour de quatre sections. La deuxième section présente la revue de la littérature. La troisième section s'intéresse à la méthodologie. Les résultats obtenus sont présentés à la quatrième section. La dernière section conclura notre travail en proposant quelques recommandations.

\section{Revue de la littérature}

\subsection{Evolution de la pauvreté au Cameroun de 1996 - 2007}

Afin d'évaluer les efforts fait en matière de réduction de la pauvreté et des inégalités, le Cameroun a réalisé successivement depuis 1996 trois enquêtes sur le plan national dit Enquête Camerounaise Auprès des Ménages (ECAM). En 1996, les rapports de la première enquête qui mesure l'évolution de la pauvreté depuis 1984 montre que près d'un camerounais sur deux était pauvre (incidence de la pauvreté estimée à 53,3\%). Cette pauvreté monétaire a reculé de 13 points entre 1996 et 2001 et restée stable sur la période 20012007. Cette stabilité du niveau de pauvreté masque cependant d'énormes disparités selon le milieu de résidence comme l'indique le graphique suivant.

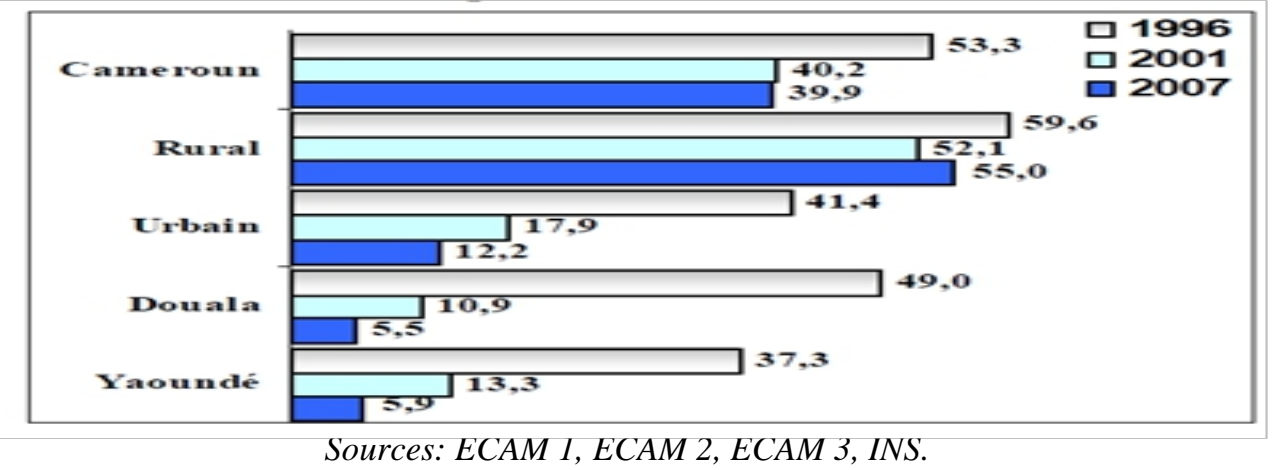

Figure 2: Evolution de l'incidence de la pauvreté entre 1996 et 2007 au Cameroun 
On remarque ainsi que si en milieu urbain l'on a assisté à une baisse importante de la paupérisation, en milieu rural la situation par rapport à 2001 s'est détériorée.

\subsection{Travaux sur l'efficience et la pauvreté \\ $>$ Définition des concepts}

Le concept de pauvreté apparemment simple, ne l'est pas pour autant. Lorsqu'un décideur politique veut engager une action de lutte contre la pauvreté, il se confronte à deux questions pratiques qui sont de savoir qui est pauvre et en quoi l'est-il ? (Diagne et al, 2005). Les réponses à ces questions sont cruciales pour l'efficacité et même pour l'effectivité de son action.

Selon l'organisation des Nations Unies (2004), la pauvreté peut être définie comme « la condition dans laquelle se trouve un être humain qui est privé de manière durable ou chronique des ressources, des moyens, des choix, de la sécurité et du pouvoir nécessaire pour jouir d'un niveau de vie suffisant et d'autres droits civils, culturels, économiques, politiques et sociaux. »

Pour la Banque mondiale (2001), " La pauvreté est synonyme de privation et de vulnérabilité. Privation d'une nutrition adéquate, de soins de santé rudimentaires, d'une éducation de base et de possibilités impossibles à saisir ».

Pour le PNUD (1998), « La pauvreté est un phénomène complexe qui désigne généralement une insuffisance de ressources et une privation de possibilités de choix et d'opportunités qui offriraient aux individus des conditions de vie décentes $»$.

Les autorités camerounaises, s'inspirant des consultations participatives en vue de l'élaboration du Document de Stratégie de Réduction de la Pauvreté considèrent que «La pauvreté est avant tout le manque de ressources matérielles ou financières pour satisfaire les besoins essentiels des individus. Au nombre de ces besoins figurent l'alimentation, le logement, les soins de santé, l'éducation, l'approvisionnement en eau potable etc. ».

Malgré tout, le consensus sur la définition de la pauvreté est loin d'être acquis. On note divers concepts de pauvreté dans la littérature : pauvreté monétaire, pauvreté subjective ${ }^{11}$, pauvreté humaine ${ }^{12}$, pauvreté infrastructurelle, pauvreté d'existence ${ }^{13}$, pauvreté psychologique, pauvreté

\footnotetext{
${ }^{11} \mathrm{~L}$ 'approche subjective repose sur l'appréciation des ménages sur leurs conditions de vie.

${ }^{12}$ La pauvreté humaine se réfère aux potentialités qu'un individu est en mesure ou non de réaliser, en fonction des possibilités qui lui sont offertes. Elle met en avant les pénuries de capacités.

${ }^{13}$ Cette approche aborde la pauvreté sous l'angle des résultats plutôt que sur des moyens à partir des conditions matérielles d'habitation (source d'éclairage du logement; sources d'énergie pour la cuisine ; types des lieux d'aisance ; matériaux des murs etc.).
} 
d'équipement, pauvreté relative à la privation, pauvreté de patrimoine etc. De ce fait, deux approches sont communément retenues, à savoir l'approche monétaire et l'approche non monétaire.

Dans cette étude, c'est l'approche monétaire de la pauvreté qui est retenue et donc sera considéré comme pauvre toute personne ayant un revenu en dessous du seuil de pauvreté que nous définirons par la suite.

La notion d'efficience économique quant 'a elle fait référence à une mesure relative de l'habilité du micro-entrepreneur du point de vue de l'optimisation du processus de production et de sa maitrise des prix des facteurs de production et du produit comparativement à l'environnement dans lequel il opère. Ainsi, selon Nabil A et Robert R (2000), une unité de production est dite efficiente si à partir du panier d'intrants qu'elle utilise, elle produit le maximum d'output possible ou si pour obtenir une quantité donnée d'output, elle utilise les plus petites quantités possibles d'intrants. La mesure du niveau d'efficience d'une unité de production permet donc de cerner si cette dernière peut accroître sa production sans pour autant consommer plus de ressources, ou diminuer l'utilisation d'au moins un intrant tout en conservant le même niveau de production.

\section{$>$ Travaux sur l'efficience et la pauvreté}

Pour Pineau (2004), la productivité est un facteur central de lutte contre la pauvreté. Il estime que la productivité est ce dont les pauvres ont besoin pour sortir de la pauvreté.

Dans la même perspective, le Centre d'étude des niveaux de vie (CSLS)14, 2003) examine la documentation économétrique limitée sur la productivité et la pauvreté et donne le mécanisme par lequel la productivité affecte la pauvreté. Il ressort que l'amélioration des capacités réduit la pauvreté en abaissant le prix des biens, lesquels coûtent moins cher à cause des gains de productivité. Elle montre également une relation inverse entre ces deux variables. C'est à dire que la pauvreté a un impact négatif sur la productivité. De nombreux travaux ont tenté de mettre en exergue l'impact d'une amélioration de la productivité et donc de l'efficience sur la pauvreté.

LACHAUD (2009) pour expliquer la féminisation de l'urbanisation de la pauvreté à Madagascar utilise une méthode paramétrique sur les données issues des enquêtes prioritaires auprès des ménages de 2001 et 2005.

Comme résultats, il constate que le ratio du taux d'efficience homme/femme a augmenté de 0,859 (2001) à 0,938 (2005) et que le taux d'efficience économique des micro-entreprises gérées par une femme a décliné de $28,5 \%$ entre 2001 et 2005 contre $21,5 \%$ pour celles ayant à la tête un homme. Au regard de ces résultats, l'auteur estime que la féminisation de

${ }^{14}$ (Centre for Study of Living Standards) Cité par Pineau (2004) 
l'urbanisation de la pauvreté est non seulement expliquée par d'autres dimensions du marché mais aussi par le déclin relatif et absolu de l'efficience économique des micro-entreprises urbaines gérées par les femmes.

Au Canada, on peut mentionner les travaux de Fluet et Lefebvre (1983) dans le secteur manufacturier. Ces auteurs ont montré que les gains de productivité conduisent à l'augmentation des prix de facteurs ou à la réduction des prix des outputs. Dans le cas de l'augmentation des prix des facteurs, les salariés et les propriétaires en sortent gagnants et la réduction des prix des outputs est bénéfique aux ménages. Enfin, ils indiquent qu'environ la moitié des gains de productivité de l'industrie manufacturière ont été transmis au reste de l'économie par une baisse des prix relatifs au cours de la période 19651980.

Pour le cas du Cameroun, nous avons la contribution significative de Justin BEM et al (2012). Ces auteurs ont utilisé la méthode non paramétrique (DEA) pour montrer que le passage à l'efficience du secteur informel contribuerait à la réduction de la pauvreté des ménages à hauteur de 19,0\%. Cependant, cette méthode (DEA) a fait l'objet de plusieurs critiques à savoir :

$>$ premièrement, la fonction frontière obtenue par cette procédure non paramétrique est déterministe. Ceci signifie que tout écart qu'une firme affiche par rapport à cette frontière est attribué à l'inefficience (aucune variation aléatoire n'est possible);

$>$ deuxièmement, la fonction frontière estimée par de telles procédures n'a aucune propriété statistique permettant de tester des hypothèses ;

$>$ enfin, la frontière estimée à l'aide de ces procédures est très sensible aux observations extrêmes, qui sont en grande partie responsables de la détermination de cette frontière.

Nous constatons au terme de cette revue de littérature que de nombreux travaux ont été réalisés dans la mesure de l'effet de l'efficience sur la pauvreté ou même dans la contribution de l'efficience de production sur le bien être des producteurs. Pour le cas des micro-entreprises camerounaises, dans l'optique d'évaluer la proportion des micro-entrepreneurs dont le revenu passerait audessus du seuil de pauvreté en situation d'efficience, nous procédons par segmentation du fait de l'hétérogénéité que constitue ces micro-entreprises tant sur la structure que sur le mode de fonctionnement. Dans la mesure où la méthode non paramétrique surévalue l'inefficience et donc sous-estime le niveau d'efficience, il sera utilisé la méthode paramétrique qui présente aussi la possibilité d'utiliser les propriétés statistiques

\section{Méthodologie et segmentation des micro-entreprises}

La méthodologie adoptée dans ce travail se décline en trois parties. Chacune est présentée dans les sections qui suivent : Estimation de la frontière 
d'efficience économique, Segmentation des micro-entreprises et l'évaluation d'impact de l'efficience sur la pauvreté.

\subsection{Estimation de la frontière d'efficience économique}

Dans ce travail, la frontière de profit est utilisée pour la mesure de l'efficience des activités de production des micro-entreprises. Ce choix s'inspire des études d'Ogunniyi, L.T(2008) qui ayant travaillé sur l'efficience a opté pour la fonction de profit qu'il entrevoit comme une extension et une formalisation des décisions de production prises par le producteur. Selon l'auteur, cette approche présente assez d'avantages eu égard au fait que l'on admet que le prix des facteurs de production et du produit ne relèvent pas du producteur mais du marché et peuvent être utilisés pour exprimer l'emploi des facteurs. Pour cette mesure, la grande difficulté réside dans le choix des facteurs devant permettre l'estimation de la frontière commune aux microentreprises sujettes à l'estimation. Pour ce choix, l'Enquête sur l'Emploi et le Secteur Informel a renseigné pour chacune des micro-entreprises, les facteurs comme : la main d'œuvre, la consommation intermédiaire, les impôts et les investissements.

La fonction de profit utilisée est de type Cobb-Douglas ceci du fait de sa flexibilité et de ses bonnes propriétés ${ }^{15}$. Ainsi, le modèle exprimé est le suivant :

$$
\pi_{i}=f(X, \beta) \exp \left(v_{i}-u_{i}\right)
$$

\section{Avec $i$ allant de 1 à $n$}

Puisque nous recherchons l'impact de l'efficience sur la pauvreté pour l'ensemble des micro-entreprises et par segment, il est important d'estimer la frontière de profit d'efficience pour chaque segment. Ainsi, la frontière de profit de la micro-entreprise i se trouvant dans le segment j s'exprimera de la manière suivante :

$$
\pi_{\mathrm{i}}=f\left(X_{i}, \beta_{j}\right) \exp \left(v_{i}-u_{i}\right)
$$

Avec $\mathrm{j}$ allant de $\mathrm{i}$ à $\mathrm{k}$ entreprise $\mathrm{i}$,

Dans cette spécification, $\pi_{i} 16$ désigne le profit mensuel de la micro-

\footnotetext{
${ }^{15}$ La fonction Cobb-Douglas est croissante et Continue, la productivité marginale des facteurs de production est décroissante, les rendements d'échelles sont constants et les conditions d'INADA sont respectées

${ }^{16}$ Ici, le profit est assimilé à l'excédent brut d'exploitation EBE
} 
$X_{i}$ est le vecteur des facteurs qui influence le profit ;

$\beta$ est le vecteur inconnu de paramètre à estimer ;

\section{Niveau d'efficience}

Ici, le niveau d'efficience est mesuré pour l'ensemble des microentreprises et dans chaque segment. On considère comme niveau d'efficience ou score d'efficience le profit de la ième micro-entreprise relativement au profit qui pourrait être obtenu par une micro-entreprise totalement efficiente en utilisant le même vecteur de facteur de production (Lachaud, 2009). Ceci est donné par la formule suivante

$\theta_{i}=\frac{\text { profit.observé }}{\text { profit.d'efficience }}=\frac{e^{x_{i} \beta+v_{i} *^{-u_{i}}}}{e^{x_{i} \beta+v_{i}}}=e^{-u_{i}}$

Où $u_{i}$ représente le terme d'inefficience.

\subsection{Facteurs explicatifs de l'efficience}

Après l'estimation du niveau d'efficience, il est nécessaire de s'intéresser aux facteurs susceptibles d'expliquer les variations de ce niveau d'efficience. Jusqu'au début des années quatre-vingt-dix du siècle passé, les études utilisaient une approche à deux étapes : soit l'estimation de la frontière stochastique dans un premier temps et une deuxième étape qui consistait à spécifier un modèle de régression mettant en relation le niveau d'efficience technique de la firme i et une série de variables socio-économiques ou autres (niveau de scolarité du gestionnaire; statut de propriété, taille de la firme, temps, etc.). Cette procédure en deux étapes a cependant été contestée puisque l'estimation des paramètres dans la seconde étape contredit une hypothèse faite dans la première, notamment l'indépendance des termes d'erreurs lors de l'estimation de la frontière. Ainsi, deux principaux types de modèles ont été proposés pour pallier à ces insuffisances. Il s'agit du modèle de Battese et Coelli et du modèle de Huang et Liu ${ }^{17}$.

Pour l'estimation des déterminants de l'efficience, nous utilisons le deuxième modèle de Battese et Coelli (1995) où ces auteurs n'ont pas tenu compte des variables d'interaction.

\subsection{Mesure de l'effet de l'efficience sur la pauvreté}

Il est question pour nous de déterminer ce qu'on gagnerait en termes de réduction de la pauvreté si toutes les micro-entreprises étaient efficientes.

\footnotetext{
${ }^{17}$ Cités par Nabil A et Robert R (2000)
} 
Selon Jocelyne Delarue et Hubert Cochet(2011), l'évaluation de l'impact d'un projet repose sur un principe simple. Ainsi, elle consiste à mesurer le différentiel entre deux situations : celle résultant de la mise en place du projet d'une part, et celle qui aurait prévalu si le projet n'avait pas été mis en place d'autre part. Il s'agit donc d'isoler dans l'indicateur d'impact la variation due au projet de celle qui aurait également eu lieu en son absence. Pour Bourguignon et Ferreira (2003) ${ }^{18}$, la particularité des méthodes d'analyse préalable est de simuler un scénario contrefactuel. Ce scénario correspond à ce qui serait passé si l'action n'avait pas été mise en œuvre. Ces derniers auteurs considèrent que le modèle doit être une simple représentation arithmétique de l'incidence de l'action envisagée sur la variable de réponse.

Soit $y=\left(y_{1}, y_{2}, \ldots y_{n}\right)$ le vecteur des revenus observés des microentrepreneurs. L'exploration des données montre que 99,4\% des microentrepreneurs déclarent ne posséder qu'une seule unité de production en 2010. Ainsi, nous faisons l'hypothèse que le revenu observé du micro-entrepreneur correspond à son profit.

Soit $\quad y^{*}=\left(y_{1}^{*}, y_{2}^{*}, \ldots . y_{n}^{*}\right)$ le vecteur des revenus obtenu en situation d'efficience des micro-entrepreneurs.

Ainsi, nous pouvons écrire :

$y_{i}^{*}=y_{i}+g_{i}$

où $g_{i}$ est le gap de revenu du micro-entrepreneur i par rapport à sa situation d'efficience. Ce terme peut être aussi interprété comme des subventions fait aux micro-entrepreneurs et permettant de compenser le montant de revenu dû à l'inefficience ou des taxes que ces derniers payent et qui réduit leur revenu potentiel.

Pour appréhender l'effet de l'efficience sur la pauvreté, nous calculons les indicateurs de pauvreté monétaire de FOSTER, GREER et THORBECKE (1984) avec le revenu observé et le revenu en situation d'efficience.

$$
\Delta P\left(y, y^{*}, p l\right)=p\left(y^{*}, p l\right)-p(y, p l)
$$

Oùpl désigne la ligne de pauvreté (seuil de pauvreté) considérée.

L'impact relatif sur la pauvreté peut donc s'exprimer comme suit :

$$
\Delta P_{r}\left(y, y^{*}, p l\right)=\frac{\Delta p\left(y, y^{*}, p l\right)}{p(y, p l)} * 100
$$

On aura pour impact absolu dans le segment $\mathrm{j}$

$$
\Delta P_{j}\left(y, y^{*}, p l\right)=p\left(y^{*}, p l\right)-p(y, p l)
$$

${ }^{18}$ Cité par Justin BEM et al (2012) 
et relatif $\Delta P_{r j}\left(y, y^{*}, p l\right)=\frac{\Delta p\left(y, y^{*}, p l\right)}{p(y, p l)} * 100$

\section{Estimation du revenu contrefactuel ou du revenu en situation d'efficience}

Étant donné que la quasi-totalité des micro-entrepreneurs déclarent n'avoir qu'une seule unité de production, le revenu contrefactuel se réduit au profit efficient. Ce profit est évalué à partir des scores d'efficience. Dans ce cas, pour une micro-entreprise $\mathrm{i}$, son revenu contrefactuel ou profit d'efficience noté $y_{i}^{*}$ est donné par :

$$
\begin{aligned}
& y_{i}^{*}=\text { profit_observé }+\left(1-\theta_{i}\right)^{*} \text { profit_observé } \\
& y_{i}^{*}=\left(2-\theta_{i}\right)^{*} \text { profit_observé }
\end{aligned}
$$

\subsection{Données de l'étude}

La base de données utilisée dans ce travail de recherche, provient de l'Enquête sur l'Emploi et le Secteur Informel (EESI) réalisée par l'Institut National de la Statistique du Cameroun en 2010. Il s'agit d'une enquête classique en 3 phases de type 1-2-3 (AFRISTAT-DIAL ,2001). La phase 1 est une enquête emploi auprès des ménages dans laquelle l'on s'intéresse aux caractéristiques démographiques, de l'emploi et du chômage des individus. Cette phase permet aussi d'identifier les chefs des établissements informels qui sont interrogés par la suite. La phase 2 est une enquête de type entreprise auprès des établissements informels, appelés «unités de production informelles » (UPI) et la troisième phase est relative à la consommation et les conditions de vie des ménages. Celle-ci n'a pas fait l'objet d'EESI, 2010.

Au cours de la première phase, $\mathbf{8} 160$ ménages ont été interrogés. Parmi ceux-ci, $\mathbf{4 7 0 5}$ unités de production informelle non agricole ont été identifiées et $\mathbf{4 5 3 8}$ ont été enquêtés avec succès à la phase 2 .

\subsection{Segmentation des micro-entreprises camerounaises}

Dans cette section, il convient de présenter la méthodologie de cette segmentation afin d'estimer son modèle.

\subsubsection{Méthodologie de segmentation}

La segmentation des micro-entreprises camerounaises se justifie par la recherche de l'homogénéité non seulement dans la structure de production mais aussi des firmes au sein desquelles l'on mesure le niveau de pauvreté. Nous nous proposons pour cela de procéder à un « clustering » par la méthode de mélange de régression finis. La méthodologie de cette méthode s'inspire 
des travaux de Mélika Ben Salem et Isabelle Bensidoun (2010). Ces auteurs ont mis sur pied dans « Emploi informels hétérogènes et segmentation du marché du travail turc » un ensemble de développement des méthodes de régression en présence d'un nombre inconnu de mélanges de distributions.

\section{a) Modèle de segmentation des micro-entreprises}

Le modèle économétrique qui permet de détecter les différents segments homogènes existant au sein des entreprises informelles camerounaises peut s'écrire intuitivement de la manière suivante :

$$
\pi_{i}=x_{i} \beta_{h}+\mu_{i}, h=1, \ldots \ldots, H
$$

i ;

Dans cette spécification, $\pi_{i}$ représente le profit de la micro-entreprise

$h$, représente la classe à laquelle appartient la micro-entreprise i ;

$\beta_{h}$, représente le vecteur des paramètres pour les individus du groupe $\mathrm{h}$;

$x_{i}$, représente les caractéristiques liées à la micro-entreprise $\mathrm{i}$.

Au regard de ce modèle, il ressort que le profit de la micro-entreprise est expliqué à la fois par les caractéristiques liées à l'entrepreneur et à la micro-entreprise. Il convient de noter que les proportions $\rho_{h}$ des différents segments ne sont pas connues en avance.

Pour ce modèle de mélange de modèle de régression, il est supposé que

$\mu_{i^{-----} \rightarrow} \rightarrow\left(0, \sigma_{\mathrm{h}}^{2}\right)$ et $\mu_{i}$ e $t \mu_{i}{ }^{\prime}$ sont non corrélés si les micro-entreprises i et i' n'appartiennent pas au même segment.

La fonction de densité correspondante à la structure de mélange de modèle de régression est la somme concave de fonction de densité dans chaque segment. Ainsi on a :

$$
f\left(\mu_{i}\right)=\sum_{i=1}^{H} \rho_{h} f_{\mathrm{h}}\left(\mu_{i}\right)
$$

C'est à dire $f\left(\mu_{i}\right)=\sum_{i=1}^{H} \rho_{h} f_{\mathrm{h}}\left(\pi_{i}-x_{i} \beta_{h}\right) \quad$ Avec $\quad \sum_{i=1}^{N} \rho_{h}=1$

Pour obtenir la loi normale centrée réduite des transformations permettent d'avoir la forme suivante :

$f\left(\mu_{i}\right)=\sum_{i=1}^{N} \rho_{h} \frac{1}{\sigma_{\mathrm{h}}} \emptyset\left(\frac{\pi_{i}-x_{i} \beta_{h}}{\sigma_{\mathrm{h}}}\right)$

Dans cette spécification, $\varnothing$ est la densité de probabilité d'une loi normale centrée réduite et la vraisemblance du modèle est ainsi donnée par :

$\mathrm{L}\left(\pi_{i} / x_{i}, \sigma\left(\sigma_{1 \ldots . .} \sigma_{h}\right), \beta_{h}, \rho_{h}\right)=\prod_{i=1}^{N} \sum_{h=0}^{H} \rho_{h} \frac{1}{\sigma_{\mathrm{h}}} \emptyset\left(\frac{\pi_{i}-x_{i} \beta_{h}}{\sigma_{\mathrm{h}}}\right)$

et en passant à la log vraisemblance on obtient :

$\ln \mathrm{L}\left(\pi_{i} / x_{i}, \sigma\left(\sigma_{1 \ldots . .} \sigma_{h}\right), \beta_{h}, \rho_{h}\right)=\sum_{i=0}^{N} \ln \left(\sum_{h=1}^{H} \rho_{h} \frac{1}{\sigma_{\mathrm{h}}} \emptyset\left(\frac{\pi_{i}-x_{i} \beta_{h}}{\sigma_{\mathrm{h}}}\right)\right)$

Selon la théorie des modèles de mélange, toutes densités de probabilité peuvent être estimées de façon convergente par un mélange de lois normales. Il convient de noter que ceci est possible sous certaines conditions de 
régularité. L’estimation des paramètres inconnus pour un nombre de segment fixé suppose qu'il existe une formulation analytique du maximum de vraisemblance. Ce qui n'est pas le cas, nous exploitons ici l'algorithme EM (Expectation-Maximisation) pour estimer les paramètres. L'utilisation de cet algorithme est fondée sur l'hypothèse selon laquelle il existe une variable d'appartenance non observée des micro-entreprises de l'échantillon aux différents segments inconnus. Cette méthode se déroule en différentes itérations. A chaque itération, la composante E de l'algorithme estime la probabilité de la micro-entreprise i d'appartenir à un segment $\mathrm{h}$ donné. Ce processus se poursuit jusqu'à ce que la valeur de la logvraisemblance n'augmente plus.

\section{b) Choix de la meilleure partition}

Le choix de la meilleure partition n'est pas toujours aisé. On note dans la littérature plusieurs outils pour juger de la partition optimale

Le premier moyen consiste à estimer le modèle pour les différents segments donnés. Ici, on s'arrête lorsqu'un segment additionnel n'apporte pas un gain additionnel en segmentation.

On peut aussi comparer les différents modèles du point de vue de la logvraisemblance, des critères d'information tels BIC (Bayésian Information Criterion) et AIC (Akaike Information Criterion). En plus de ces critères, nous utilisons la statistique EN (Entropy Normalized)

$$
\begin{aligned}
& \text { Elle est donnée par } E N_{k}=1-\frac{E_{K}}{N \ln (K)} \\
& \text { Où } \quad E_{K}=-\sum_{i=1}^{N} \sum_{k=1}^{K} t_{i k} \ln (K)
\end{aligned}
$$

Avec $t_{i k}$ représentant la probabilité que l'individu i appartient à la classe $\mathrm{k}$;

$\mathrm{N}$ le nombre d'observation et $\mathrm{K}$ le nombre de classes de la partition. La statistique ENk apporte plus de précision quant à la distribution de probabilité entre les individus au sein des différents segments. Ainsi, la partition en K classe est préférable pour tout $\mathrm{k}$ appartenant à $1 \ldots \mathrm{K}$, si ENk a la plus grande valeur possible.

Pour l'estimation de ce modèle, nous faisons recours au logiciel R où nous utilisons la fonction « Regmix EM » du package « Mixtools ».

\section{b) Résultats du modèle de segmentation et interprétation}

Pour l'estimation de ce modèle, nous avons mis en œuvre l'algorithme Expectation-Maximisation (EM) pour les partitions de 2,3, 4 segments. L'arrêt à 4 segments se justifie par l'émiettement des tailles des segments 
additionnels. Pour le choix de la meilleure partition, nous utilisons le tableau suivant présentant les critères ICL (Integrated Complete-data Likelihood), $\mathrm{AIC}, \mathrm{BIC}$, et $\mathrm{EN}_{\mathrm{k}}$.

Tableau 1:Critères d'information pour le choix de la meilleure partition

\begin{tabular}{|r|r|r|r|}
\hline & K=2 & K=3 & K=4 \\
\hline AIC & $\mathbf{- 2 4 5 2 8 , 7 6}$ & $\mathbf{- 2 3 9 7 4 , 1 4}$ & $\mathbf{- 2 3 4 7 9 , 6 2}$ \\
\hline BIC & $\mathbf{- 2 4 5 9 9 , 3 8}$ & $\mathbf{- 2 4 0 8 3 , 2 8}$ & $\mathbf{- 2 3 6 2 7 , 2 8}$ \\
\hline ICL & $\mathbf{- 2 4 5 9 9 , 0 2}$ & $\mathbf{- 2 4 0 8 2 , 3 8}$ & $\mathbf{- 2 3 6 2 6 , 1 7}$ \\
\hline ENk $_{\mathbf{~}}$ & $\mathbf{0 , 8 8}$ & $\mathbf{0 , 7 1}$ & $\mathbf{0 , 6 4}$ \\
\hline
\end{tabular}

Sortie de $R$ et noc calculs avec les données de l'EESI, INS, 2010

Au regard de tableau 1, une partition de 4 segments parait meilleur du fait qu'elle minimise les valeurs de ICL, AIC, BIC. Cependant, le critère ENK montre que la partition en 2 segments apporte plus de précision dans l'affectation des individus. Ainsi, nous allons choisi une partition en deux classes.

\section{i) Précision dans l'affectation des micro-entreprises aux différents segments}

Ici, nous allons utiliser les probabilités d'appartenance des individus aux différents segments. Une partition est considérée meilleure si elle permet d'obtenir une segmentation où les individus sont classés avec des probabilités de classement élevées. Ceci nous permet d'obtenir le tableau 2 suivant.

Tableau 2: Classe de probabilité d'affectation et segmentation

\begin{tabular}{|l|l|l|l|l|l|l|l|}
\hline Tik & {$[\mathbf{1 , 0 - 0 , 9}[$} & {$[\mathbf{0 , 9 - 0 , 8}[$} & {$[\mathbf{0 , 8 - 0 , 7}[$} & {$[\mathbf{0 , 7 - 0 , 6}[$} & {$[\mathbf{0 , 6 - 0 , 5}[$} & {$[\mathbf{0 , 5 - 0 , 4}[$} & Total \\
\hline K=2 & $\mathbf{4 3 3 9}$ & $\mathbf{8 1}$ & $\mathbf{4 6}$ & $\mathbf{4 3}$ & $\mathbf{2 8}$ & $\mathbf{0}$ & $\mathbf{4 5 3 7}$ \\
\hline $\mathrm{K}=\mathbf{3}$ & $\mathbf{2 4 5 3}$ & $\mathbf{1 4 1 3}$ & $\mathbf{2 9 2}$ & $\mathbf{2 0 8}$ & $\mathbf{1 6 7}$ & $\mathbf{4}$ & $\mathbf{4 5 3 7}$ \\
\hline $\mathrm{K}=\mathbf{4}$ & $\mathbf{1 6 9 2}$ & $\mathbf{1 1 2 6}$ & $\mathbf{9 8 4}$ & $\mathbf{4 3 8}$ & $\mathbf{2 7 2}$ & $\mathbf{2 5}$ & $\mathbf{4 5 3 7}$ \\
\hline
\end{tabular}

Sortie de $R$ et nos calculs avec les données de l'EESI, INS, 2010

Ce tableau2 permet de constater que le modèle spécifié est un bon modèle pour cette segmentation. En effet, la proportion des micro-entreprises affectée avec une plus grande précision $(0,9<=$ Prob $<=1)$ est très élevée pour chacune des trois partitions : Il est de 95,63\% Pour la partition en 2 segments et de $54,07 \%$ pour la partition en 3 segments et $37,29 \%$ pour celle en 4 segments.

Compte tenu de ce tableau, il est clair que la segmentation en deux classes est appropriée.

\section{ii) Description et caractérisation des différents segments}

Dans cette partie, nous examinons les différents segments ou classes suivant les caractéristiques de positionnement de la micro-entreprise dans le marché, les caractéristiques du promoteur et les caractéristiques de l'unité de production.

\section{Premier segment}


Ce segment représente $92,4 \%$ de l'ensemble des micro-entreprises.

Considérant les caractéristiques du marché, il convient de mentionner que la plupart des micro-entrepreneurs de cette classe exerce dans le commerce en détail (31,5\%) suivi de l'agro-alimentaire $(18,0 \%)$. Les ménagers et les particuliers constituent leurs principaux clients et représentent ainsi près de $88,0 \%$ de l'ensemble des clients. Ces micro-entrepreneurs en grande partie n'ont pas de numéro de contribuable $(92,0 \%)$ et seulement 3,8\% ont essayé avec succès d'obtenir une carte de contribuable. De plus, leur approvisionnement est fait majoritairement pas des petites structures constituées des petites entreprises commerciales, des petites entreprises non commerciales.

S'agissant des caractéristiques liées aux micro-entreprises, il ressort que ces dernières réalisent un chiffre d'affaire mensuel moyen de 204,96 MFCFA et un profit mensuel moyen de 60,9156 MFCFA.

Quant aux promoteurs, on remarque que la majeure partie de ces derniers n'a que le niveau primaire $(43,2 \%)$ et très peu ont fait le supérieur $(5,3 \%)$. De plus, il faut remarquer que c'est une classe où on rencontre plus de femmes promoteurs $(56,0 \%)$. Ces caractéristiques peuvent ainsi justifier la faiblesse de leurs revenus constatés.

\section{Deuxième segment.}

Dans le deuxième segment, le chiffre d'affaires mensuel moyen et le profit sont respectivement de 1505,01 MFCFA et 393,9478 MFCFA. Ces chiffres d'affaire et profits représentent presque 7 fois ceux de la première classe.

Dans cette classe, bien que la proportion des acteurs n'ayant pas de numéro de contribuable reste élevée $(85,5 \%)$, on remarque que $6,8 \%$ de ces acteurs ont une carte de contribuable.

S'agissant du niveau d'instruction, on constate que le pourcentage des promoteurs ayant un niveau supérieur s'est amélioré. Ce pourcentage est de $7 \%$ contre $5,3 \%$ pour le segment précédent. De plus, la plus par des promoteurs sont les hommes $(67,5 \%)$.

Une fois présenté la méthodologie et la caractérisation des différents segments rencontrés au sein des micro-entreprises camerounaises, il nous importe de déterminer l'impact de l'efficience sur le niveau de vie des microentrepreneurs à travers la frontière stochastique de profit.

\section{Résultat de l'étude}

\subsection{Scores ou niveau d'efficience des micro-entreprises}

Il ressort de nos analyses que le score moyen global d'efficience est de $60 \%$. Ce score est de 58,7\% dans le premier segment et $75 \%$ dans le second segment. Ainsi, dans l'ensemble, les micro-entreprises auraient pu réaliser 
$40 \%$ de plus de profit qu'ils ne réalisent actuellement avec les quantités de facteurs utilisées.

\subsection{Interprétation des résultats d'analyse des facteurs explicatifs d'efficience}

Tableau 3: Facteurs explicatifs du niveau d'efficience (premier segment)

\begin{tabular}{|c|c|c|c|c|c|c|}
\hline $\begin{array}{c}\text { Terme d'inefficience } \\
\left(u \_e\right)\end{array}$ & & Coef, & $\begin{array}{c}\text { niveau moyen } \\
\text { d'efficience }\end{array}$ & Std, Err, & $T$ & $P>t$ \\
\hline \multirow[t]{2}{*}{ Sexe } & Masculin & Réf & 0,582 & & & \\
\hline & Féminin & $-0,1628436$ & 0,5 & 0,0189193 & $-8,61$ & 0 \\
\hline \multirow[t]{4}{*}{ Ancienneté } & Moins de 3ans & Réf & 0,521 & & & \\
\hline & entre 3 et 5 ans & $-0,0580791$ & 0,549 & 0,02458 & $-2,36$ & 0,018 \\
\hline & entre 5 et 8 ans & $-0,1000342$ & 0,574 & 0,0285174 & $-3,51$ & 0 \\
\hline & plus 8 ans & $-0,0395985$ & 0,539 & 0,0248893 & $-1,59$ & 0,112 \\
\hline \multirow[t]{4}{*}{ Age } & Moins de 26 ans & Réf & 0,511 & & & \\
\hline & entre 27 et 34 & $-0,0540804$ & 0,552 & 0,0241076 & $-2,24$ & 0,025 \\
\hline & entre 35 et 44 & $-0,0939078$ & 0,559 & 0,026045 & $-3,61$ & 0 \\
\hline & plus 45 ans & $-0,0064767$ & 0,518 & 0,0282618 & $-0,23$ & 0,819 \\
\hline \multirow[t]{5}{*}{ Niveau d'instruction } & sans niveau & Réf & 0,448 & & & \\
\hline & Primaire & $-0,035551$ & 0,521 & 0,1626014 & $-0,22$ & 0,827 \\
\hline & secondaire général & $-0,0448895$ & 0,541 & 0,1626052 & $-0,28$ & 0,783 \\
\hline & sec technique & $-0,0162266$ & 0,547 & 0,163867 & $-0,1$ & 0,921 \\
\hline & Supérieur & $-0,3250505$ & 0,588 & 0,1359362 & $-2,39$ & 0,017 \\
\hline \multirow[t]{3}{*}{$\begin{array}{c}\text { Existence des } \\
\text { salariés }\end{array}$} & Oui & Réf & 0,594 & & & \\
\hline & Non & $-0,0137141$ & 0,532 & 0,1056165 & $-1,13$ & 0,097 \\
\hline & Oui & Réf & 0,537 & & & \\
\hline \multirow[t]{2}{*}{ obtention des prêts } & Non & 0,0335685 & 0,535 & 0,0181048 & 1,85 & 0,064 \\
\hline & Oui & Réf & 0,604 & & & \\
\hline \multirow[t]{2}{*}{ membre association } & Non & $-0,1109903$ & 0,534 & 0,0492432 & $-2,25$ & 0,014 \\
\hline & Oui & Réf & 0,565 & & & \\
\hline numéro contribuable & Non & $-0,4168966$ & 0,533 & 0,0315631 & $-13,21$ & 0 \\
\hline \multirow[t]{3}{*}{ Type de local } & bien installées & Réf & 0,587 & & & \\
\hline & passablement inst, & $-0,0392365$ & 0,507 & 0,0202067 & $-1,94$ & 0,052 \\
\hline & sans local fixe & $-0,0486631$ & 0,54 & 0,0237875 & $-2,05$ & 0,041 \\
\hline \multirow[t]{10}{*}{ Région } & Douala & Réf & 0,576 & & & \\
\hline & Yaoundé & $-0,0792019$ & 0,564 & 0,0294033 & $-2,69$ & 0,007 \\
\hline & Adamaoua & $-0,1894453$ & 0,517 & 0,0521222 & $-3,63$ & 0 \\
\hline & Centre & $-0,2033077$ & 0,52 & 0,0431119 & $-4,72$ & 0 \\
\hline & Est & $-0,2234331$ & 0,553 & 0,0529986 & $-4,22$ & 0 \\
\hline & Extrême-nord & $-0,2330571$ & 0,491 & 0,0333645 & $-6,99$ & 0 \\
\hline & Littoral & $-0,178571$ & 0,57 & 0,0394411 & $-4,53$ & 0 \\
\hline & Nord & $-0,2420808$ & 0,494 & 0,0365112 & $-6,63$ & 0 \\
\hline & Nord-ouest & $-0,2251607$ & 0,461 & 0,0405946 & $-5,55$ & 0 \\
\hline & Ouest & $-0,2373415$ & 0,545 & 0,0336641 & $-7,05$ & 0 \\
\hline
\end{tabular}




\begin{tabular}{|c|c|c|c|c|c|c|}
\hline & Sud & $-0,0890567$ & 0,489 & 0,0561758 & $-1,59$ & 0,113 \\
\hline & Sud-ouest & $-0,1245024$ & 0,52 & 0,0470804 & $-2,64$ & 0,008 \\
\hline & Urbain & Réf & 0,556 & & & \\
\hline Milieu & Rural & $-0,0903542$ & 0,502 & 0,0222677 & $-4,06$ & 0 \\
\hline \multirow[t]{4}{*}{ Type d'emploi } & Auto-emploi & Réf & 0,53 & & & \\
\hline & Non salarié & $-0,0790866$ & 0,563 & 0,0324806 & $-2,43$ & 0,015 \\
\hline & Salarié & $-0,2552996$ & 0,604 & 0,0696229 & $-3,67$ & 0 \\
\hline & Mixte & $-0,0400401$ & 0,634 & 0,0974261 & $-0,41$ & 0,681 \\
\hline \multirow[t]{4}{*}{ Branche } & Industrie & Réf & 0,479 & & & \\
\hline & Commerce & $-0,2949425$ & 0,565 & 0,0227763 & $-12,95$ & 0 \\
\hline & Service & $-0,1422521$ & 0,56 & 0,0221514 & $-6,42$ & 0 \\
\hline & Patron & Réf & 0,582 & & & \\
\hline Statut & Travailleur & $-0,1501131$ & 0,532 & 0,0900659 & $-1,67$ & 0,096 \\
\hline \multirow[t]{4}{*}{ principal fournisseur } & Grande structure & Réf & 0,572 & & & \\
\hline & Ménagers/particulier & $-0,3216252$ & 0,55 & 0,0407958 & $-7,88$ & 0 \\
\hline & petite structure & $-0,2148054$ & 0,52 & 0,0262673 & $-8,18$ & 0 \\
\hline & non concerné & $-0,5533901$ & 0,57 & 0,0344976 & $-16,04$ & 0 \\
\hline \multirow[t]{3}{*}{ principal client } & Grande structure & Réf & 0,574 & & & \\
\hline & Ménagers/particulier & $-0,1415203$ & 0,529 & 0,0752585 & $-1,88$ & 0,06 \\
\hline & petite structure & $-0,1413174$ & 0.585 & 0,0790513 & $-1,79$ & 0,074 \\
\hline cons & Constante & 12,16244 & & 0,1945703 & 62,51 & 0 \\
\hline
\end{tabular}

Source : sortie de stata et nos calculs avec les données de l'EESI, INS, 2010

\section{a) Facteurs explicatifs du niveau d'efficience dans le premier segment i) Facteurs liés au promoteur}

Il ressort du tableau 3 que les élasticités du terme d'inefficience par rapport à l'âge, au statut et au sexe du micro-entrepreneur sont significatives respectivement au seuil de $1 \%, 5 \%$ et $10 \%$. Ainsi, l'inefficience des microentreprises camerounaises est influencée par l'âge, le statut et par le sexe du micro-entrepreneur. L'efficience des micro-entreprises dépend du sexe .En effet, comparativement aux hommes, les femmes sont moins efficientes. Le niveau moyen d'efficience des micro-entreprises dirigées par les hommes dépasse celui des femmes de $8,2 \%$.

En ce qui concerne l'âge, les résultats nous permettent de remarquer que le niveau d'efficience croit avec cette variable. Il est de 51,1\%, 55,2\%, $55,9 \%$ respectivement pour les moins de 26 ans, les 26 à 34 ans et les 35 à 44 ans. On observe un retournement du niveau d'efficience pour les plus de 45 ans. En effet, on est passé de $55,9 \%$ pour les 35 à 44 ans à $51,8 \%$ pour les plus de 45 ans. On constate ainsi que lorsque le micro-entrepreneur vieilli, il est moins efficient. Ceci peut s'expliquer par la nature individuelle de la gestion des micro-entreprises dont l'enthousiasme et l'habilité dégradent avec le temps. Les micro-entrepreneurs ayant un statut de patron sont plus efficients que ceux ayant un statut de travailleur pour compte propre. On note un écart de 5,0\%. Ceci se justifie par le fait que le patron contraint ses salariés pour des résultats précis et le faisant, il accroît son niveau d'efficience. Quant au niveau 
d'instruction, on est un peu surpris du fait que seul le niveau supérieur influence l'inefficience des promoteurs. Malgré cela, on peut remarquer que le niveau d'efficience augmente avec le niveau d'instruction. On est passé de $44.8 \%$ pour les sans niveau à $58.8 \%$ pour les micro-entrepreneurs de niveau supérieur.

\section{ii) Facteurs de positionnement}

Les élasticités du terme d'inefficience par rapport à : l'appartenance ou non à une association professionnelle, à la branche d'activité, à la possession ou non d'un numéro de contribuable, au principal fournisseur de la micro-entreprise et au milieu sont significatives au seuil de $1 \%$. Ces différentes variables ont une influence positive sur le niveau d'efficience. En effet en référence aux micro-entrepreneurs qui appartiennent à une association, ceux qui n'en font pas partir sont moins efficients. Il en résulte un écart de niveau de 7,0\%. Ceci pourrait être justifié par le fait que les microentrepreneurs qui appartiennent à une association partagent leur savoir-faire entre eux. On remarque que l'obtention ou non de prêt ne change pas grande chose sur le niveau d'efficience des micro-entrepreneurs (ce niveau est de $53,7 \%$ pour ceux ayant obtenus des prêts contre $53,5 \%$ pour ceux qui n'ont pas obtenus). Ceci se comprend du fait que très peu de micro-entrepreneurs sollicitent de crédit.

Pour les branches d'activités, les résultats montrent que les microentreprises camerounaises en 2010 présentent les niveaux d'efficience suivant classés par ordre : la branche commerce $(56,5 \%)$, la branche service $(56,0 \%)$ et la branche industrie $(47,9 \%)$. Les micro-entrepreneurs qui ont un numéro de contribuable surpassent Ceux qui n'ont pas de 3,2\%. Ceci montre que la détention d'un numéro de contribuable oblige le micro-entrepreneur à fournir plus d'effort dans la gestion et lui permet aussi de gagner de gros marchés. L'influence liée à la provenance des ressources est significative. On retrouve par ordre d'efficience, ceux qui sont fournis par les grandes structures $(57,2 \%)$, ceux qui utilisent leurs propres ressources $(57,0 \%)$, ceux qui ont pour fournisseurs des ménagers/particuliers $(55,0 \%)$ et ceux qui sont fournis par les petites structures $(52,0 \%)$. Quant à la position géographique, il est à noter que l'élasticité du terme d'inefficience est globalement significative par rapport aux régions au seuil de 5\%. Les niveaux d'efficience par région et classés par ordre se présentent comme suit : Douala (57,6\%), Littoral $(57,0 \%)$, Yaoundé (56,4\%), Est (55,3\%), Ouest( 54,5\%), Centre (52,0\%),Sud-ouest $(52,0 \%)$, Adamaoua $(51,7 \%)$, Nord $(49,4 \%)$, Extrême-nord $(49,1 \%)$, Sud $(48,9 \%)$, Nord-ouest $(46,1 \%)$. Ces résultats paraissent plus ou moins explicables dans la mesure où les deux grandes villes du Cameroun présentent un certain nombre d'atouts incontestables pour le développement des activités de survie. Ceci est vrai pour la région de l'ouest. 


\section{iii) Facteurs liés à la micro-entreprise}

S'agissant des caractéristiques de la micro-entreprise, on remarque que l'ancienneté de celle-ci est significative par rapport au terme d'inefficience au seuil de $1 \%$. Le niveau d'efficience augmente avec l'ancienneté de la microentreprise. Ce niveau est de 52,1\%,54,9\%, 57,4\% respectivement pour les micro-entreprises ayant moins de 3 ans, celles de 3 à 5 ans et celles de 5 à 8 ans. A partir de 8 ans et plus, les résultats montrent une baisse du niveau d'efficience. On est passé de $57,4 \%$ pour les 5 à 8 ans à $51,8 \%$ pour les 8 ans et plus. Concernant l'existence ou non de la main d'œuvre salarié, on remarque que cette variable influence le niveau d'efficience comme le statut du micro-entrepreneur. En effet, comparativement aux micro-entreprises qui n'ont pas de salariés, ceux qui ont les salariés surpassent ces derniers de $6,2 \%$. Ceci est peut-être dû au fait que les salariés sont obligés de produire des résultats fixés par leur patron.

\section{b) Facteurs explicatifs du niveau d'efficience dans le deuxième segment}

Tableau 4: Régression de l'inefficience sur les facteurs explicatifs de l'efficience dans le deuxième segment

\begin{tabular}{|c|c|c|c|c|c|c|}
\hline $\begin{array}{c}\text { Terme } \\
\text { d'inefficience } \\
\left(\mathbf{u} \_ \text {e }\right)\end{array}$ & & Coef, & $\begin{array}{c}\text { niveau moyen } \\
\text { d'efficience }\end{array}$ & Std, Err, & $\mathbf{t}$ & $P>t$ \\
\hline & Masculin & réf & $\mathbf{0 , 7 3 5}$ & & & \\
\hline Sexe & Feminin & $-0,0487527$ & 0,744 & 0,0516736 & $-0,94$ & 0,346 \\
\hline \multirow[t]{4}{*}{ Anciénneté } & moins de 3 ans & réf & $\mathbf{0 , 7 3}$ & & & \\
\hline & 3--5 ans & $-0,0574875$ & $\mathbf{0 , 7 3 3}$ & 0,0610369 & $-0,94$ & 0,347 \\
\hline & 5--8 ans & $-0,1026291$ & 0,736 & 0,0710373 & $-1,44$ & 0,15 \\
\hline & plus 8 ans & $-0,086616$ & 0,75 & 0,0631441 & $-1,37$ & 0,171 \\
\hline \multirow[t]{4}{*}{ Age } & moins de 26 ans & réf & 0,741 & & & \\
\hline & 26--34 ans & $-0,0170685$ & $\mathbf{0 , 7 3 3}$ & 0,0635636 & $-0,27$ & 0,788 \\
\hline & $35-44$ ans & $-0,0818316$ & 0,736 & 0,0706858 & $-1,16$ & 0,248 \\
\hline & plus de 45 ans & $-0,003306$ & 0,745 & 0,0784419 & $-0,04$ & 0,966 \\
\hline \multirow[t]{6}{*}{$\begin{array}{c}\text { Niveau } \\
\text { d'instuction } \\
\end{array}$} & sans niveau & réf & $\mathbf{0 , 7 5 7}$ & & & \\
\hline & primaire & $-0,2321567$ & $\mathbf{0 , 7 3 7}$ & 0,2794882 & $-0,83$ & 0,407 \\
\hline & secondiaregeneral & $-0,2153731$ & $\mathbf{0 , 7 3 5}$ & 0,2782636 & $-0,77$ & 0,44 \\
\hline & $\begin{array}{l}\text { secondaire } \\
\text { technique }\end{array}$ & $-0,2351139$ & $\mathbf{0 , 7 3 9}$ & 0,2816136 & $-0,83$ & 0,404 \\
\hline & Supreieur & $-0,13105$ & 0,745 & 0,2918268 & $-0,45$ & 0,654 \\
\hline & Oui & réf & $\mathbf{0 , 7 3 7}$ & & & \\
\hline \multirow[t]{2}{*}{$\begin{array}{c}\text { Existence } \\
\text { des salariés }\end{array}$} & Non & $-0,5635383$ & $\mathbf{0 , 7 3 7}$ & 0,2503818 & $-2,25$ & 0,025 \\
\hline & Oui & réf & 0,74 & & & \\
\hline \multirow[t]{2}{*}{$\begin{array}{l}\text { obtention } \\
\text { des prêts }\end{array}$} & Non & $-0,0158784$ & $\mathbf{0 , 7 3 6}$ & 0,0479036 & $-0,33$ & 0,741 \\
\hline & Oui & réf & 0,748 & & & \\
\hline \multirow[t]{2}{*}{$\begin{array}{c}\text { membre } \\
\text { association }\end{array}$} & Non & $-0,000589$ & $\mathbf{0 , 7 3 6}$ & 0,0859591 & $-0,01$ & 0,995 \\
\hline & Oui & réf & 0,72 & & & \\
\hline
\end{tabular}




\begin{tabular}{|c|c|c|c|c|c|c|}
\hline $\begin{array}{c}\text { numéro } \\
\text { contribuable }\end{array}$ & Non & $-0,2270118$ & $\mathbf{0 , 7 4 2}$ & 0,0664136 & $-3,42$ & 0,001 \\
\hline \multirow[t]{3}{*}{ Type de local } & bien installées & réf & $\mathbf{0 , 7 4 5}$ & & & \\
\hline & passablement inst, & $-0,0298794$ & $\mathbf{0 , 7 4 7}$ & $\mathbf{0 , 0 2 6 8 3 1}$ & $-1,11$ & 0,266 \\
\hline & sans local fixe & $-0,0645489$ & $\mathbf{0 , 7 2 3}$ & 0,0668307 & $-0,97$ & 0,335 \\
\hline \multirow[t]{12}{*}{ Région } & Douala & réf & $\mathbf{0 , 7 3 9}$ & & & \\
\hline & Yaoundé & $-0,0568506$ & $\mathbf{0 , 7 2 9}$ & 0,0671715 & $-0,85$ & 0,398 \\
\hline & Adamaoua & $-0,0219255$ & $\mathbf{0 , 7 1 3}$ & 0,133136 & $-0,16$ & 0,869 \\
\hline & Centre & $-0,0003673$ & $\mathbf{0 , 7 4 5}$ & 0,1291951 & 0 & 0,998 \\
\hline & Est & $-0,1094469$ & $\mathbf{0 , 7 5 2}$ & 0,1291853 & $-0,85$ & 0,398 \\
\hline & Extrême-nord & $-0,1147669$ & $\mathbf{0 , 7 7 2}$ & 0,1036401 & $-1,11$ & 0,269 \\
\hline & Littoral & $-0,0388836$ & $\mathbf{0 , 7 3 4}$ & 0,1044598 & $-0,37$ & 0,71 \\
\hline & Nord & $-0,060019$ & 0,776 & 0,0916401 & $-0,65$ & 0,513 \\
\hline & Nord-ouest & $-0,0263589$ & $\mathbf{0 , 7 0 5}$ & 0,1023279 & $-0,26$ & 0,797 \\
\hline & Ouest & $-0,1614661$ & $\mathbf{0 , 7 3 6}$ & 0,099055 & $-1,63$ & 0,104 \\
\hline & Sud & $-0,0909888$ & 0,715 & 0,1401536 & $-0,65$ & 0,517 \\
\hline & Sud-ouest & $-0,0056698$ & 0,678 & 0,1239581 & $-0,05$ & 0,964 \\
\hline \multirow[t]{4}{*}{$\begin{array}{c}\text { Type } \\
\text { d'emploi }\end{array}$} & Auto-emploi & réf & $\mathbf{0 , 7 3 9}$ & & & \\
\hline & non salarié & $-0,0248899$ & $\mathbf{0 , 7 3}$ & 0,03147 & $-0,79$ & 0,429 \\
\hline & Salarié & $-0,1850322$ & $\mathbf{0 , 7 2 1}$ & 0,044608 & $-4,15$ & $\mathbf{0}$ \\
\hline & Mixte & $-0,110492$ & 0,771 & $\mathbf{0 , 0 5 7 2 3 3}$ & $-1,93$ & $\mathbf{0 , 0 5 4}$ \\
\hline \multirow[t]{4}{*}{ Branche } & Industrie & réf & $\mathbf{0 , 7 4 4}$ & & & \\
\hline & Commerce & $-0,3462686$ & $\mathbf{0 , 7 4 6}$ & 0,066901 & $-5,18$ & 0 \\
\hline & Service & $-0,0441023$ & $\mathbf{0 , 7 2 4}$ & 0,0596441 & $-0,74$ & 0,46 \\
\hline & Patron & réf & $\mathbf{0 , 7 3 8}$ & & & \\
\hline Statut & Travail & $-0,377104$ & $\mathbf{0 , 7 3 7}$ & 0,2371607 & $-1,59$ & 0,113 \\
\hline \multirow[t]{4}{*}{$\begin{array}{c}\text { principal } \\
\text { fournisseur }\end{array}$} & grande struicture & réf & $\mathbf{0 , 7 1 8}$ & & & \\
\hline & Ménager/particulier & $-0,2176148$ & 0,741 & 0,1161841 & $-1,87$ & 0,062 \\
\hline & petite structure & $-0,0341162$ & 0,742 & 0,063288 & $-0,54$ & 0,59 \\
\hline & non concerné & $-0,353162$ & $\mathbf{0 , 7 6 2}$ & 0,0874457 & $-4,04$ & 0 \\
\hline \multirow[t]{2}{*}{ Milieu } & Urbain & réf & 0,735 & & & \\
\hline & Rural & $-0,0475312$ & 0,744 & 0,0672403 & $-0,71$ & 0,48 \\
\hline \multirow[t]{3}{*}{$\begin{array}{c}\text { principal } \\
\text { client }\end{array}$} & grande struicture & réf & & & & \\
\hline & Ménager/particulier & $-0,3245875$ & & 0,1264514 & $-2,57$ & 0,011 \\
\hline & petite structure & $-0,2796206$ & & 0,1343346 & $-2,08$ & 0,038 \\
\hline _cons & constante & 13,0497 & & $\mathbf{0 , 2 0 9 7 9 6}$ & 62,2 & $\mathbf{0}$ \\
\hline
\end{tabular}

Source : sortie de stata et nos calculs avec les données de l'EESI, INS, 2010

L'observation du tableau 4 permet de constater que les différentes caractéristiques (Positionnement, liées au promoteur, à la structure) n'influencent presque pas le niveau d'efficience dans le deuxième segment. En effet, pour toutes ces variables, seule la branche d'activité, la possession d'un numéro de contribuable, l'existence des salariés, le type d'emploi et le principal fournisseur et client ont une influence significative sur le niveau d'efficience. Ce résultat est surprenant dans la mesure où on sait que l'instruction d'un promoteur élargit son champ d'intervention et donc contribue à améliorer ses performances. Il en est de même pour l'âge surtout quand on sait que le promoteur accumule de l'expérience avec le temps. 


\section{c) Facteurs explicatifs du niveau d'efficience dans l'ensemble}

En considérant l'ensemble des micro-entreprises, il ressort que tous les facteurs qui influencent le niveau d'efficience dans le premier segment restent valables lorsqu'on considère les micro-entreprises dans l'ensemble. Il faut cependant remarquer que le niveau d'instruction des micro-entrepreneurs n'a pas d'influence sur l'efficience. Ce constat ne corrobore pas avec les différentes analyses faites précédemment. Puisqu' 'il est établi que l'alphabétisation contribue à élargir le champ de compétence des microentrepreneurs et donc augmente leur niveau d'efficience.

\subsection{Les Indices FGT et impact de l'efficience sur la pauvreté.}

Le seuil de pauvreté retenu ici est celui issu de l'ECAM3. Il est de 269443 FCFA soit 22453,58FCFA par mois. Pour le calcul des indicateurs monétaires de la pauvreté, nous utilisons le revenu du micro-entrepreneur approximé par le profit comme indicateur de niveau de vie au lieu de la consommation. Car l'enquête EESI n'avait pas pour objectif de mesurer les conditions de vie. Il est à noter que dans cette étude, nous supposons sans nuire à la généralité que le micro-entrepreneur n'est pas chef de ménage et consomme tout seul son revenu. Ceci permet de ramener l'impact de l'efficience au niveau individuel. En effet il est question pour nous de voir si un micro-entrepreneur peut vivre décemment de son activité. Nous allons mesurer cet impact pour l'ensemble des micro-entreprises et dans chaque segment. Les résultats sont présentés dans le tableau 5 :

Tableau 5 : Impact potentiel sur la pauvreté

\begin{tabular}{|c|c|c|c|c|}
\hline Indicateur & $\begin{array}{l}\text { Indices } \\
\text { FGT }\end{array}$ & $\begin{array}{l}\text { Situation initiale en } \\
\%\end{array}$ & $\begin{array}{l}\text { Situation d'efficience } \\
\text { en } \%\end{array}$ & $\begin{array}{l}\text { Variation en } \\
\%\end{array}$ \\
\hline \multirow{3}{*}{$\begin{array}{l}\mathrm{P}_{\mathrm{O}}=\operatorname{taux} \mathrm{de} \\
\text { pauvreté }\end{array}$} & Ensemble & 42,5 & 31,6 & $-10,9$ \\
\hline & Segment 1 & 45,1 & 33,6 & $-11,5$ \\
\hline & Segment 2 & 10,5 & 7.5 & $-3,1$ \\
\hline \multirow{3}{*}{$\begin{array}{l}\mathrm{P}_{1}=\text { profondeur } \\
\text { de la pauvreté }\end{array}$} & Ensemble & 24,4 & 15,9 & -8.5 \\
\hline & Segment1 & 25,7 & 16,8 & -8.9 \\
\hline & Segment 2 & $\mathbf{7 , 0}$ & 4,7 & $-2,3$ \\
\hline \multirow{3}{*}{$\begin{array}{l}\mathrm{P}_{2}=\text { sévérité de la } \\
\text { pauvreté }\end{array}$} & Ensemble & 17,2 & 10,2 & -7 \\
\hline & Segment 1 & 18,2 & 10,8 & $-7,4$ \\
\hline & Segment 2 & 5,2 & 3,4 & $-1,8$ \\
\hline
\end{tabular}

Source : Sortie de DAD et nos calculs avec les données de l'EESI, INS, 2010.

$\mathrm{Au}$ regard du tableau5, il ressort qu'en considérant les microentreprises camerounaises dans l'ensemble près de $42,0 \%$ enregistrent un profit inférieur au seuil de pauvreté selon ECAM3. A l'efficience, cette incidence passe à $31,6 \%$. Soit une baisse de $10,9 \%$. Cela voudrait dire que la situation d'efficience permet de sortir $10,9 \%$ des micro-entrepreneurs dans la situation de pauvreté. Du fait de l'hétérogénéité de ses micros unités de production, la situation d'efficience affectera différemment les entrepreneurs 
du secteur. Il ressort que le premier segment est celui qui regroupe presque tous les acteurs qui ne parviennent pas à vivre décemment de leurs activités. En effet, à la situation initiale on dénombre près de $45,1 \%$ des acteurs qui vivent en dessous du seuil de pauvreté. Le constat est intéressant pour les acteurs du deuxième segment où seulement 10,5\% sont pauvres. Le profit efficient permettrait à $11,5 \%$ des entrepreneurs du deuxième segment de vivre avec un revenu au moins égal au seuil de pauvreté. Dans le deuxième segment, la situation d'efficience permettrait à $3,5 \%$ des pauvres de devenir non pauvres. Il faut remarquer que l'effet est non négligeable sur la profondeur et la sévérité de la pauvreté aussi bien dans l'ensemble que dans les différents segments comme le montre le tableau ci-haut.

\section{Conclusion et recommandations}

Cette étude avait pour objectif de mesurer la contribution de l'efficience de production dans la réduction de la pauvreté des microentrepreneurs et d'identifier les facteurs explicatifs du niveau d'efficience, ceci à travers la segmentation des micro-entreprises. Pour y arriver, nous avons eu recours aux données de l'Enquête sur l'Emploi et le Secteur Informel, réalisée en 2010 par l'Institut National de la Statistique.

Pour la segmentation, il ressort que le tissu micro-entrepreneurial camerounais présente deux segments. Le premier segment représente $92,4 \%$ de l'ensemble des micro-entreprises. Il est caractérisé par une faiblesse non négligeable de revenu. En effet, les acteurs de ce segment réalisent un chiffre d'affaire moyen mensuel de 204,96 MFCFA et un profit mensuel moyen de $60,19156 \mathrm{MFCFA}$. Il est à remarquer que la grande partie des microentrepreneurs de ce segment n'ont pas de numéro de contribuable (92,0\%).Le deuxième segment quant à lui représente $7,6 \%$ des micro-entreprises totales. Ce segment est formé des micro-entrepreneurs dont le chiffre d'affaire et le profit sont considérable .En effet, ils sont respectivement de 1505,01 MFCFA et 393,9478 MFCFA en moyenne et par mois. On y trouve plus d'hommes promoteurs $(67,5 \%)$ que de femmes $(32,5 \%)$.

L'estimation de la frontière stochastique de profit nous a permis de mettre effectivement en évidence la présence du terme d'inefficience et du terme aléatoire aussi bien pour l'ensemble des micro-entreprises que dans chaque segment. Ceci justifie donc l'usage de la méthode paramétrique utilisée. Ainsi l'utilisation de la méthode non paramétrique qui consiste à affecter toute la différence entre le profit observé et le profit efficient à l'inefficience n'est donc pas appropriée.

Concernant l'impact de l'efficience sur la pauvreté, nous avons remarqué que la remise à l'efficience des micro-entreprises permettrait une réduction de la pauvreté non négligeable des promoteurs du secteur. Il s'est avéré que dans l'ensemble, $42,5 \%$ des micro-entrepreneurs camerounais 
tiraient de leurs activités un profit inférieur au seuil de pauvreté (qui est de 269443 FCFA par ans suivant ECAM III) Parmi ceux-ci, 10,9\% passeraient au-dessus du seuil de pauvreté en situation d'efficience. En s'intéressant aux segments, on note que le premier segment regroupe presque tous les pauvres $\mathrm{du}$ tissu micro-entrepreneurial camerounais avec un taux de pauvreté de 45,1\% . Parmi les $10,9 \%$ des micro-entrepreneurs qui verront leurs revenus supérieurs aux seuils de pauvreté à l'efficience, $11.5 \%$ appartiennent au premier segment et $3.1 \%$ au deuxième segment. Il convient de mentionner que Les micro-entreprises du deuxième segment qui restent pauvres à l'efficience sont des micro-entreprises jeunes. En effet, elles ont toutes moins de 3 ans d'ancienneté. Nous comprenons donc que l'amélioration de l'efficience des activités de production des micro-entrepreneurs à un effet bénéfique sur leur niveau de vie.

L'analyse des facteurs explicatifs du niveau d'efficience montre que dans le premier segment, l'efficience est effectivement influencée par les facteurs de positionnement, les facteurs liés aux promoteurs et les facteurs liés à la micro-entreprise. Pour les facteurs de positionnement, on note que le niveau d'efficience augment avec : l'appartenance de la micro-entreprise à une association de son domaine d'activité, la possession d'un numéro de contribuable, le fournisseur de la structure, le milieu et la région d'activité, Pour les facteurs liés aux promoteurs, on note l'influence positive de : l'âge, statut, sexe et du niveau d'instruction du micro-entrepreneur. Concernant les facteurs liés à la micro-entreprise, on a pu constater que l'efficience est influencé par l'ancienneté et l'existence de la main d'ouvre. Il convient tout de même de noter qu'il s'est avéré que lorsqu'une micro-entreprise est trop vieille, elle devient moins efficiente. Ceci pouvant être expliqué par la nature individuelle de la gestion des micro-entreprises.

Dans le deuxième segment, le constat est surprenant. En effet de toutes nos variables postulées, seule la possession d'un numéro de contribuable, le fournisseur et le client de la structure, l'existence des salariés et la branche d'activité de la micro-entreprise influencent positivement le niveau d'efficience.

Pour les micro-entreprises dans l'ensemble, on retrouve les résultats similaires à ceux du premier segment excepté quelques facteurs qui au niveau global n'influencent plus le niveau d'efficience. Il s'agit du niveau d'instruction. Ce constat est fort surprenant dans la mesure où il est reconnu que le niveau d'instruction améliore la compétence de l'entreprise.

$\mathrm{Au}$ vue de l'importance de l'efficience sur la réduction de la pauvreté et dans l'optique d'aider les micro-entrepreneurs qui n'ont pas pu gagner un revenu supérieur au seuil de pauvreté en situation d'efficience, les deux recommandations suivantes peuvent être formulées : 
$>$ encourager la création des villages d'activités et des associations ou regroupement des micro-entrepreneurs ayant des activités similaires pour favoriser l'amélioration du niveau d'efficience. Dans ce cas, les petites et moyennes micro-entreprises (micro-entreprise du premier segment) des régions du Sud, du Nord, du Nord-ouest, de l'Extrêmenord du fait de leur faible niveau d'efficience méritent une attention particulière ;

$>$ promouvoir l'alphabétisme et les ateliers de recyclage.

En effet, nous avons remarqué pour le premier segment où la pauvreté sévit beaucoup plus que le niveau d'efficience augmentait avec le niveau d'instruction. Ainsi les efforts pour soutenir le secteur informel doivent prendre en compte non seulement l'alphabétisation des micro-entrepreneurs mais aussi l'organisation des formations dans les différents domaines de l'activité économique en termes de recyclage.

Dans l'ensemble, nous avons constaté que les micro-entreprises du deuxième segment sont les plus efficientes. Elles présentent les caractéristiques proches de celles des entreprises du secteur formel. Ainsi, dans l'optique de les permettre de gagner des grands marchés, il est important de faciliter leur acheminement vers le secteur formel. Dans ce cas, nous trouvons approprié la stratégie envisagée dans le Document de Stratégie pour la Croissance et l'Emploi (DSCE) qui porte sur l'organisation de leurs activités en très petites entreprises (TPE) à travers : (i) une réglementation simple sur la fiscalité ; (ii) la facilitation de l'enregistrement administrative y compris la sécurité sociale; (iii) la formation dans le but d'aider ces acteurs à mieux suivre leurs activités par la tenue d'une comptabilité légère ; (iv) l'aide à l'installation et aux financements.

\section{References:}

1. Baden.S (1997), Economic reform and poverty: A gender analysis bridge, rapport n0:50, University sof Sussex;

2. Banque Mondiale (2001), "Combattre la pauvreté », Rapport sur le développement dans le monde, ${ }^{\circ}$ 2001-01, Paris, ESKA, XIV-381p ;

3. Ben Salem M. et Bensidoun I. (2010), "Heterogeneous informal jobs and segmentation of the Turkish labour market (emplois informels hétérogènes et segmentation du marché du travail turc)", CEE, France;

4. Coelli, T.J, Rao, D.S.P, Battese, G.E., (1998). An introduction to efficiency and productivity analysis, Kluwer Academic Publishers Boston;

5. Coelli, T.J., (1996). A guide to DEAP, version 2.1: A data Envelopment analysis (computer) program, CEPRO, working paper96/08, Departement of Econometrics, University of New England, Armidale; 
6. Coelli, T.J., (1996).A guide to FRONTIER, version 4.1: A computer program for frontier production function estimation, CEPA, Working paper 96/07, Departement of Econometrics, University of New England, Armide;

7. Diagne O. D., Faye O. et Faye S., (2005), « le noyau dur de la pauvreté au Sénégal » cahier de recherché PMMA - PEP, Dakar, Novembre 2005, $45 \mathrm{p}$

8. Document Stratégique de la Croissance et l'Emploi, 2009, DSCE 2009 ;

9. Farrrell, M.J, (1957), The measurement of productive efficiency, Journal of the royal Statistical Society, Series A, CXX, Part3,253-290;

10. Fluet, C \&Lefebvre, P. (1983), « Gain de productivité globale, prix relatifs et rémunération des facteurs dans les industries manufacturières au Québec », l'Actualité économique 59(4) ,651668 ;

11. Foster J., Greer J et Thorbecke E., (1984), «Aclass of decomposable poverty measures $\gg$. Econometrica52, 761-767;

12. INS (2002), Evolution de la pauvreté entre 1996 et 2001, résumé exécutive ;

13. INS (2002), Evolution de la pauvreté entre 1996 et 2001, résumé exécutive ;

14. INS (2007), Document de méthodologie générale, Troisième Enquête camerounaise

15. Jocelyne Delarue et Hubert Cochet, « Proposition méthodologique pour l'évaluation des projets de développement agricole. L'évaluation systémique d'impact », Économie rurale, 323 | 2011, 36-54 ;

16. Justin Bem, NguetseTegoum P.J, TcheekoSamo T.M, Essoh J (2012) "Production efficiency of non-agricultural informal sector and poverty reduction in Cameroun", Cahier de recherché PMMA 11864 ;

17. Lachaudjean-pierre (2009), « Profit, Efficience et genre des microentreprises urbaines à Madagascar. Existe-il une courbe de Kuznets ?, DT/148/2009 Groupe d'Economie Lare-Efi du développement, Université Montesquieu-Bordeaux IV ;

18. MANGA, T. A. N et. EPO, N. B. (2009), «Pauvreté Multidimensionnelle au Cameroun une alternative par l'Analyse en Composantes Principales », p.http://economics.ca/2009/papers/0729.pdf.

19. Nabil Amara et Robert Romain, Mesure de l'efficacité technique : Revue de la littérature, 2000 ;

20. Ogunniyi L T , « Profit Efficiency among Cocoyam Producers in Osun State », Nigeria Department of Agricultural Economics and 
Rural Devlopment, Ladoke Akintola University of Technology, Ogbomosho, Nigeria

21. Pierre-Olivier Pineau (2004), «La productivité, moyen de lutte contre la pauvreté : Étude d'une micro institution au Pérou », Université de Victoria 